\title{
Hardware sequencing of inflatable non-linear actuators for autonomous soft robots
}

Benjamin Gorissen*, Edoardo Milana, Arne Baeyens, Eva Broeders, Jeroen Christiaens, Klaas Collin, Dominiek Reynaerts*, Michael De Volder*

Dr. B. Gorissen, E. Milana, A. Baeyens, E. Broeders, J. Christiaens, K. Collin, Prof. M. De Volder, Prof. D. Reynaerts

Department of Mechanical Engineering, KU Leuven and Flanders Make, Celestijnenlaan 300, 3001 Leuven, Belgium

E-mail: benjamin.gorissen@kuleuven.be,dominiek.reynaerts@kuleuven.be

Prof. M. De Volder

Institute for Manufacturing, Department of Engineering, University of Cambridge, 17 Charles Babbage Road, Cambridge CB3 OFS, UK

E-mail:mfld2@cam.ac.uk

Keywords: soft robotics, hardware intelligence, sequencing, inflatable actuators, nonlinear actuators, soft actuators, autonomous robots

ABSTRACT

Soft robotic systems are an interesting alternative for classic rigid robots in applications requiring interaction with living organisms or delicate objects. Elastic inflatable actuators are one of the preferred actuation mechanisms for soft robots since they are intrinsically safe and soft. However, these pneumatic actuators each require a dedicated pressure supply and valve to drive and control their actuation sequence. Because of the relatively large size of pressure supplies and valves compared to electrical leads and electronic controllers, tethering pneumatic soft robots with multiple degrees of freedom is bulky and unpractical. This article describes a new approach to embed hardware intelligence in soft robots where multiple actuators are attached to the same pressure supply, and their actuation sequence is programmed by the interaction between non-linear actuators and passive flow restrictions. We show how to model this hardware sequencing, and demonstrate it on a 8 degree-of-freedom walking robot where 
each limb comprises two actuators with a sequence embedded in their hardware. Our robot is able to carry pay loads of $800 \mathrm{gr}$ in addition to its own weight, and is able to walk at travel speeds of 3 body lengths per minute, without the need for complex on-board valves or bulky tethers.

Since the early 60 s, most industrial robots have been designed as a series of rigid links and joints that are driven by localized actuators. In a more recent approach to robotics, soft members and actuators that have infinite degrees of freedom are combined into so-called soft robots [1]. Because of their soft and compliant nature, these robots inherently struggle with tasks that need accurate positioning or high forces. However, they excel at tasks that require dexterity [2], safety [3], or compliance to an unknown environment or freeform object [4]. During the past three decades, impressive advances have been made in the design and fabrication of soft robotic actuators, and a wide variety of actuation principles have been used to drive these systems [1, 5-9]. The focus of this research is on actuators that are powered through pneumatic inflation [10], which are attractive for soft robotics because of their simplicity. These actuators typically only consist of an elastic inflatable structure that converts a pressurized fluidic input to an expansion [11-14], contraction [15-18], bending [19-22] or twisting [23-26] output deformation. Further, these actuators can be combined to create complex soft robots with applications in medicine [27-30], automation [20, 31-33] and biomimetic locomotion [10, 34-36].

Because of their simplicity and ability to create complex motions, soft inflatable actuators are one of the most attractive solutions for driving future soft robots. However, to power these actuators, they each require a pressure supply tube and a pressure control valve. Because these are larger than electrical leads and control circuits, the peripheral requirements for inflatable actuators rapidly become prohibitively bulky for multiple degree-of-freedom (DoF) robots. Challenges in tethering are therefore a key factor inhibiting wider breakthroughs of fluidic soft robotics. To address this challenge, some remarkable advances have been made in miniature 
pressure supplies $[37,38]$ and valves $[39,40]$, some of which have recently been integrated in soft actuators [41]. However, these systems still require a chemical or electrical power supply, and often result in bulky systems for multiple degree-of-freedom robotic systems [42]. These challenges are summarized in Figure 1: In most of the current soft robots, separate pressure supply tubes are used for each inflatable actuator along with off-board valves and control systems (Figure 1A). Alternatively, on-board valves are used to replace multiple pneumatic tethers by electrical tethers in combination with fewer pressure supply tubes or an on-board pressure generator (Figure 1B) [43]. Alternatively, the on-board valves can be controlled pneumatically [27], or passive fluidic valves are used that convert the pressure input to a fluidic actuation sequence $[40,44]$. However, on-board valves remain voluminous and stiff, reducing load capacity and shape compliance of the overall robot.

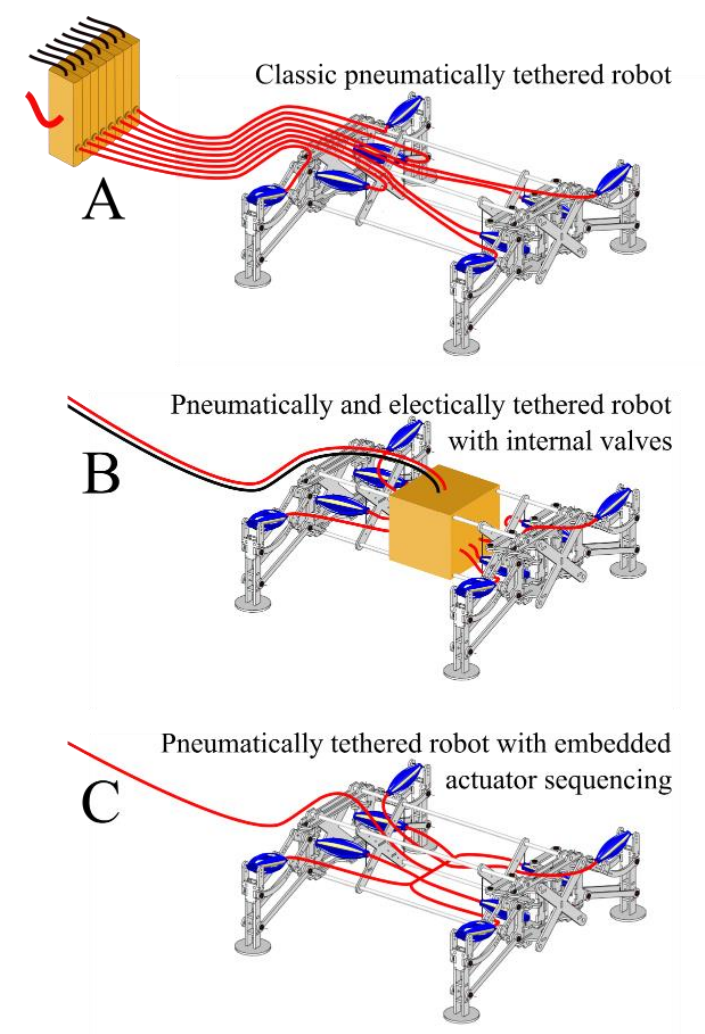

Figure 1. Tethering concepts of soft robots where each degree-of-freedom is actuated using a dedicated controlled fluidic input (A), that can be reduced to a single fluidic tether and internal valves with electrical tethers (B). By tuning the non-linear properties of the actuators with intermediate flow restrictions this can be further reduced to a single fluidic supply tube, without the need for internal valves (C). 
Here we suggest an alternative hardware sequencing approach, where no active nor passive valves are needed to create a programmable actuator sequence (Figure 1C). Hardware sequencing is achieved by tuning the non-linear properties of the inflatable actuators and the pressure drop over passive flow restrictions to a predetermined single periodic pressure input. In our approach, two pneumatic components are needed: a pneumatic inflatable actuator with a clear peak and valley in their pressure versus volume (PV) characteristics, and flow restrictions with tunable pressure losses. Peak-and-valley PV characteristics often occur in inflatable elastic actuators as a result of the changing stiffness of the elastic membrane when being inflated, with a typical example being party balloons [45]. Here we use a peak-and-valley actuator, consisting of an inner latex tube constrained by a slitted surrounding polyethylene braid [46, 47], as detailed in SI. These actuators longitudinally contract when pressurized as depicted in Figure 2, and our measurements of the actuator's PV characteristic clearly shows a peak-and-valley PV curve. This characteristic is caused by the ballooning of the inner latex tube, while the valley and the consequent pressure rise originates from contact between inner balloon and outer braid after a certain volume has been reached. As has been shown by Overvelde et al. [46] the characteristics of these actuators in terms of peak and valley pressures can be tuned by altering the length of the inserted latex tube and the length of the surrounding braid, where other deformation types are possible when altering the slit pattern of the outside braid [47]. 
A) Schematic Design

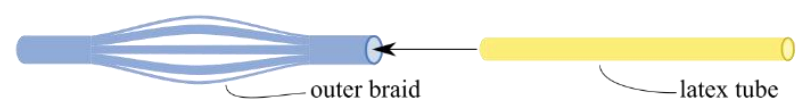

B) Schematic Actuation

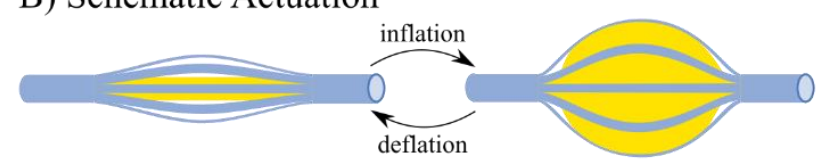

C) Pressure/Contraction vs. Volume Characteristic

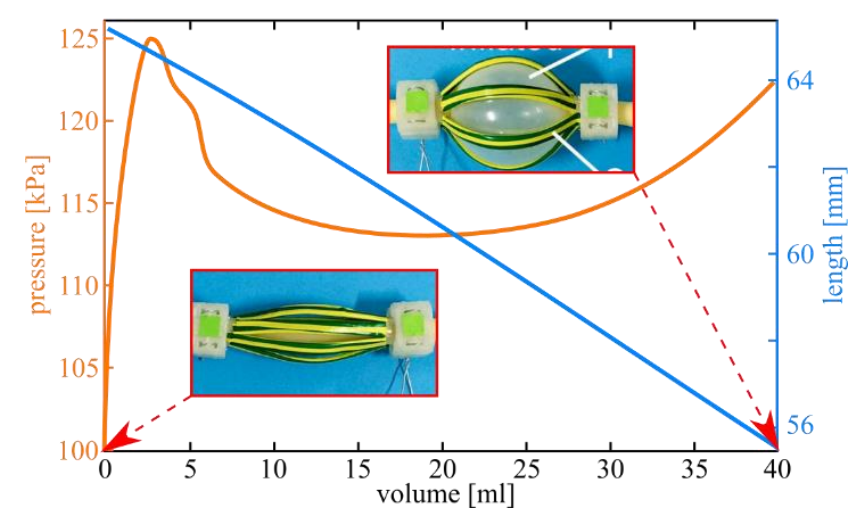

Figure 2. (A) Schematic design of an elastic inflatable actuator consisting of a highly flexible inner latex tube that is inserted into a slitted outer braid. (B) Upon inflation, the volume increase is transformed into an axial contraction of the actuator. (C) Pressure and length versus input volume, recorded using a syringe pump and simultaneously recording pressure using a pressure transducer and a deformation measurement using subsequent camera images.

When connecting two of these contracting actuators, with PV characteristics shown on Figure $\mathbf{3 A}$, in parallel to a pressure supply, their inflation and deflation can be elegantly analyzed by mirroring the PV curve of actuator 2 along its pressure axis and offsetting it in volume with an amount equal to the input volume of the total system, $V$, in accordance to [45]. At this total volume, equilibrium occurs at the intersection of both curves with an equilibrium pressure, $p_{12}(V)$. This is shown on Figure $3 \mathrm{C}$ for two input volumes $\left(V^{*}\right.$ and $\left.V^{* *}\right)$. The internal volume of each individual actuator can also be deduced from these figures, as indicated with $v_{1}(V)$ and $v_{2}(V)$. For a continuous value of $V, p_{12}(V)$ is depicted on Figure $3 \mathrm{~B}$, together with the corresponding internal volumes of actuator 1 and 2, indicated by colored area. From this graph, it can be concluded, that until volume $\mathrm{V}^{* *}$ is reached, the vast majority of the total volume is channeled to actuator 1 . After $\mathrm{V}^{* *}$, actuator 2 starts to inflate until both actuators are inflated at volume $\mathrm{V}^{* * *}$. The corresponding actuator deformations are shown on inset images of Figure 
3B. It is essential to note that the lower peak pressure of actuator 1 is inhibiting the inflation of actuator 2 until a critical volume is reached, and that the peaks and valleys in the combined PV curve correspond to the original peaks and valleys of the individual actuators. The same analysis can be repeated for actuator deflation, resulting in the same PV curve and deformations that are in symmetry with inflation. For simplicity, we will denote this actuation sequence where actuator 1 inflates before actuator 2, and actuator 2 deflates before 1 as $12 \mid 21$. This sequence can be programmed in the hardware of the robot by making the peak and valley pressures of actuator 1 lower than those of actuator 2 .
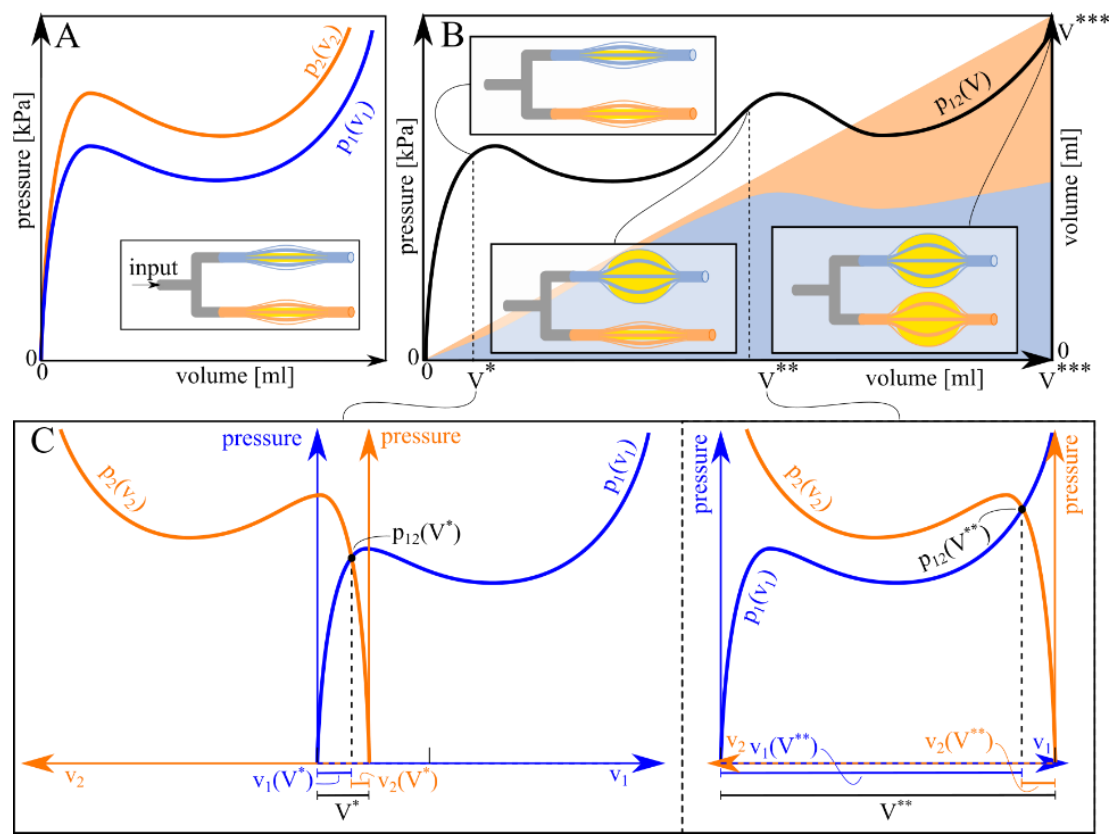

Figure 3. (A) 12|21 sequence originating from two actuators with offset PV characteristics connected in parallel. (B) Global PV characteristics from inflation this system. The volume distribution between actuators in terms of the total input volume is indicated by the colored areas and is shown on inset images. (C) The global characteristics can be analyzed by flipping the PV curve of actuator 2 along its pressure axis and translating it along the horizontal axis with the total input volume of the system. This is done here for a total input volume $\boldsymbol{V}^{*}$ and $\boldsymbol{V}^{* *}$.

The other possible sequence, $12 \mid 12$, is harder to achieve as the peak pressure of actuator 2 needs to be higher than the peak pressure of actuator 1 while the valley pressure of actuator 1 needs to be higher than the valley pressure of actuator 2 [48]. Instead, this paper suggests a simpler solution, where a pressure drop, in the form of a passive flow restriction between the two 
actuators instigates the same effect, as is shown on Figure 4 for two identical actuators. For now, and for ease of analysis, a fixed pressure drop $(\Delta p)$ is used. However in reality, this pressure drop is dependent on fluid flow [49], an aspect which will be detailed later on. Essentially, the flow resistance raises the PV curve of actuator 2 while inflating and lowers it while deflating, as perceived by actuator 1 (index *). This is depicted on Figure 4B with rising arrows for inflation and descending arrows for deflation. The corresponding total system characteristics are shown on Figure 4CD, exhibiting the 12|12 sequence, with corresponding actuator deformation shown on inset pictures, which is the result of the same analysis as explained in Figure 3C. Although the presented theory is a simplification of reality, as it is assuming fixed $\Delta$ p's and monotonous inflation/deflation (which is not always the case [46]), it can be considered a rough design tool to dimension the flow restrictions and actuators in order to achieve a certain sequence that can be then fine-tuned using a more detailed analytical approach.
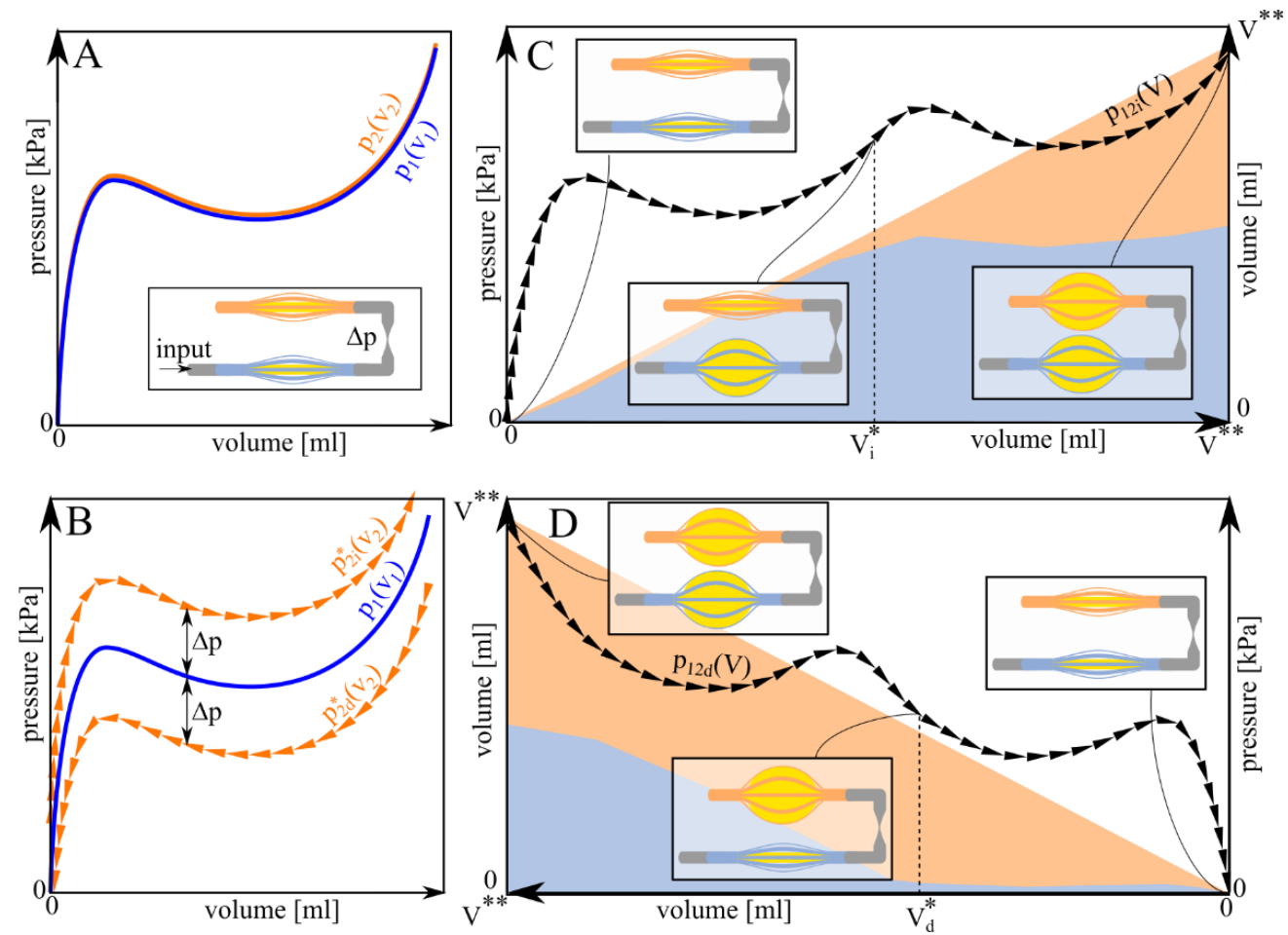

Figure 4. (A) 12|12 sequence created when a flow restriction $(\Delta \boldsymbol{p})$ is placed between two identical actuators. (B) A flow restriction offsets the PV characteristic of actuator 2 relative to actuator 1 , by $\Delta \boldsymbol{p}$ upwards during inflation (rising arrows) and downwards during deflation (descending arrows). (C) The resulting system characteristics during inflation and (D) during deflation. 
For the analytical modelling, we will make use of filling $(N)$ as the driving parameter, which can be thought of as the number of air molecules that are present in the system. As such, we can shift from volume to filling, using the ideal gas law:

$N_{i}=\frac{p_{i} V_{i}}{R T} \quad, \quad i=1 \ldots n$

Where index ' $i$ ' denotes the different actuators that are present in the system, $R$ is the ideal gas constant, $T$ the absolute temperature of the system and $p_{i}, V_{i}$ are the pressure and volume inside the actuator, which are also related to each other using the actuators PV characteristic: $p_{i}\left(V_{i}\right)$. To capture the highly nonlinear response of our soft actuators, this analysis relies on recorded PV characteristics of actuator prototypes. The actuators with preceding flow restrictions can be placed in series or in parallel, where no fluid losses are assumed at junctions:

$\sum_{\text {incoming }} \dot{N}_{J}=\sum_{\text {outgoing }} \dot{N}_{J}$

where the dot indicates a derivative in time. As we consider incompressible fluid flow, which is acceptable for moderate flow speeds, the presence of a flow restriction can be modelled using the Darcy-Weisbach equation [49]:

$p_{\text {out }}-p_{\text {in }}=f \cdot \frac{L}{D} \cdot \frac{\rho\langle v\rangle^{2}}{2}$,

where $f$ is the Darcy friction factor of the restriction, $L$ its length, $D$ its hydraulic diameter, $\rho$ the density of the fluid, $p_{\text {out }}-p_{\text {in }}$ the pressure drop over the flow restriction and $\langle v\rangle$ the average speed of fluid flow, which can be converted to filling flux using (1):

$\dot{N}=\frac{\langle p\rangle A}{R T}\langle v\rangle$,

where $A$ is the cross sectional area of the restriction and $\langle p\rangle$ the average pressure inside the flow restriction, which in this paper has been taken to be:

$$
\langle p\rangle=\frac{p_{\text {out }}+p_{\text {in }}}{2} .
$$

This set of equations make it possible to quasi-statically simulate the response of an arbitrary 
system to a pressure input, where for each incremental time step, all equations are in equilibrium. This has been implemented on a two actuator system with a supply tube length $L_{1}$ and interconnecting tube length $L_{2}$, as shown on Figure $\mathbf{5 A}$, where PV curves are measured on a prototype actuator. A pressure block pulse ( $89 \mathrm{kPa}$ for 2 seconds) has been applied as input, where supply tube length is constant $\left(L_{1}=1 \mathrm{~m}\right)$ and two lengths of interconnecting tube have been simulated $\left(L_{2}=0.3 \mathrm{~m}, 8 \mathrm{~m}\right)$, both with an inner diameter of $2 \mathrm{~mm}$ and assumed constant Darcy friction $(f=0.04)$. Simulation results are depicted on Figure 5B and Figure S1, clearly showing the 12|12 sequence, with the inflation rate of the second actuator decreasing if $\mathrm{L}_{2}$ increases.
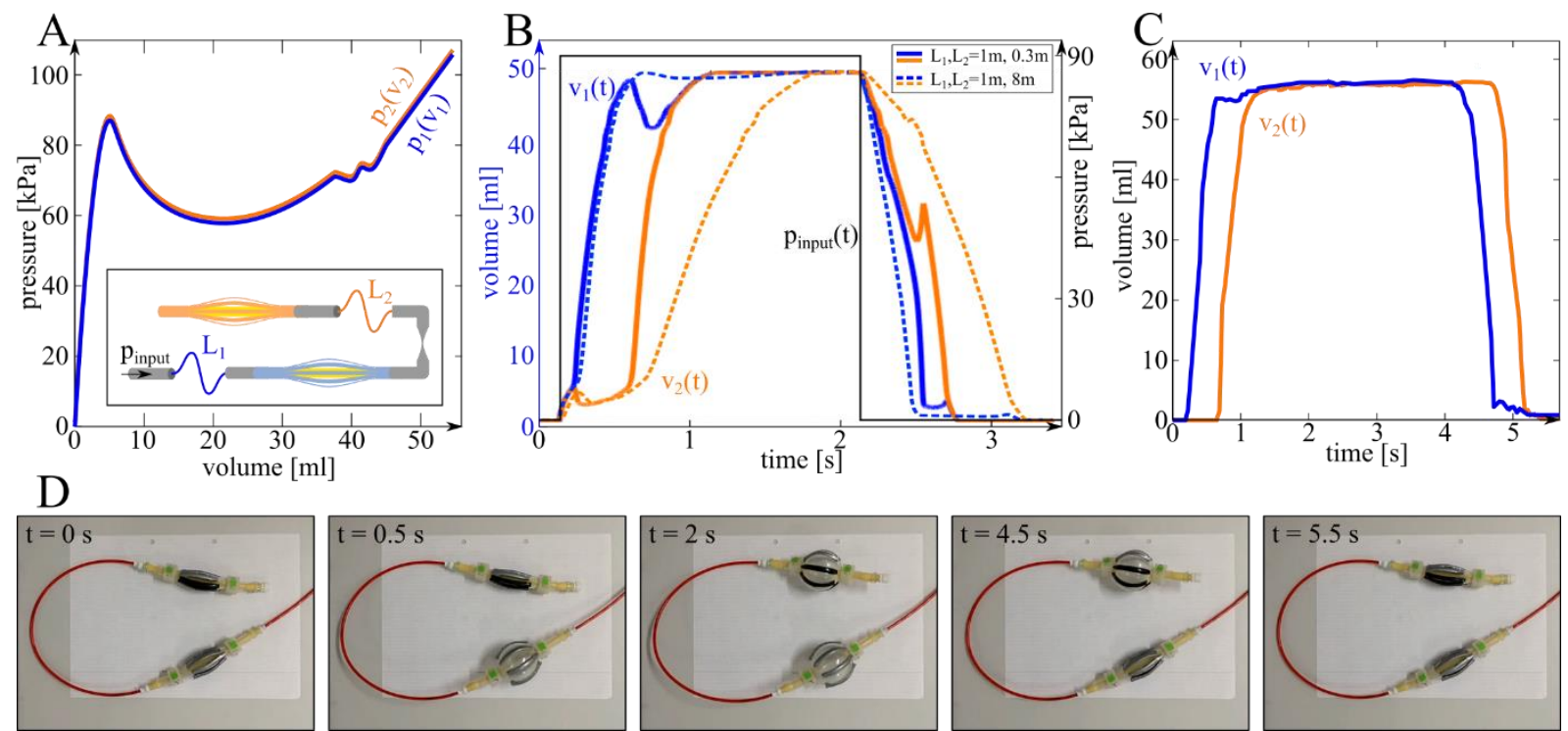

Figure 5. (A) Modeled two-actuator system that incorporates the measured PV curve of a prototype actuator, where the actuators are placed in series with a supply tube length $\boldsymbol{L}_{\mathbf{1}}$ and an interconnecting tube length $\boldsymbol{L}_{2}$. (B) Modeled dynamic response to a block pressure pulse as a function of individual actuator volume, where $\boldsymbol{L}_{\mathbf{1}}$ is fixed to $1 \mathrm{~m}$ and two values of $\boldsymbol{L}_{\mathbf{2}}$ are used: $0.3 \mathrm{~m}$ (solid lines) and $8 \mathrm{~m}$ (dashed lines). The real response of this two-actuator system has been captured as a function of individual actuator volume (C), using video recording (D and Movie S1).

To validate the presented theory to control actuator sequencing with a simple flow restriction, the two actuator system of Figure 5A $\left(L_{1}=1 m, L_{2}=0.3 m\right)$ has been fabricated and subject to a block pulse pressure input. The response of this system can be seen on Figure 5D, which clearly shows a 12|12 sequence (see also Movie S1). To validate our model, markers on the end 
points of the actuators are tracked by video software, which can be used to plot the volume versus time characteristics using the previously registered length versus volume relation (Figure 2, blue), as shown on Figure 5C. In contrast to the analytical model, this prototype system shows a more discrete sequence, as made visible on Figure S2. This discrepancy can be explained by the assumptions that have been made in the model: incompressible fluid flow, constant Darcy friction factor and ignoring the dynamics of the actuators themselves. Further, it has been observed that the addition of flow restrictions reduces the sensitivity of the actuator sequence to variations in individual PV curves, which are hard to avoid due to variability of the production process [46].

The proposed sequencing technique can be broadened to multiple actuators, as demonstrated in Figure S3, where generally the actuation sequence can be described as follows. The order of actuation during inflations is determined by the magnitude of the pressure peaks: actuators with lower peak pressures will inflate first. The order of deflation is determined by the magnitude of the pressure valleys: actuator with highest valley pressures will deflate first. As the magnitudes of peak and valley pressure of individual actuators are directly linked to the optima of the combined system (figure 3B), further sequencing is possible by separating the magnitudes of the optima in distinct pressure bands. The magnitudes of these optima values can be tuned by either actuator characteristics or by changing the preceding flow restriction, where the fixed $\Delta \mathrm{p}$-approach can be used a first rough approximation, that can be fine-tuned using the presented analytical model.

The ability to drive the actuators in a $12 \mid 12$ sequence is of huge importance, as it enables to create motion asymmetry which is needed for various types of locomotion. For instance, the knee and hip joint of humans are roughly sequenced in 12|12 when walking. To demonstrate the elegance the presented hardware sequencing theory, a tetrapod walking robot has been built, as shown on Figure 6A. This soft robotic walker comprises four legs, where each leg is actuated using two nonlinear actuators. A schematic overview of this walker can be seen on Figure $6 \mathrm{~B}$ 
and Figure S4A. In this figure, blue colored actuators control the vertical motion of the feet, while orange colored actuators control their horizontal motion. The mechanism that converts actuator contraction to foot motion, is displayed on Figure 6C for vertical motion, where a parallelogram structure is used to keep the feet always horizontal to ground (see linkage mechanism on figure S4B). Figure 6D and figure S4C show the cantilever system that is used to transfer actuator contraction to horizontal motion. When the blue and orange actuators are driven in a 12|12 sequence, the robot legs continuously perform a downward, backward, upward, forward motion (see figure 6E and MovieS2); which enables walking.
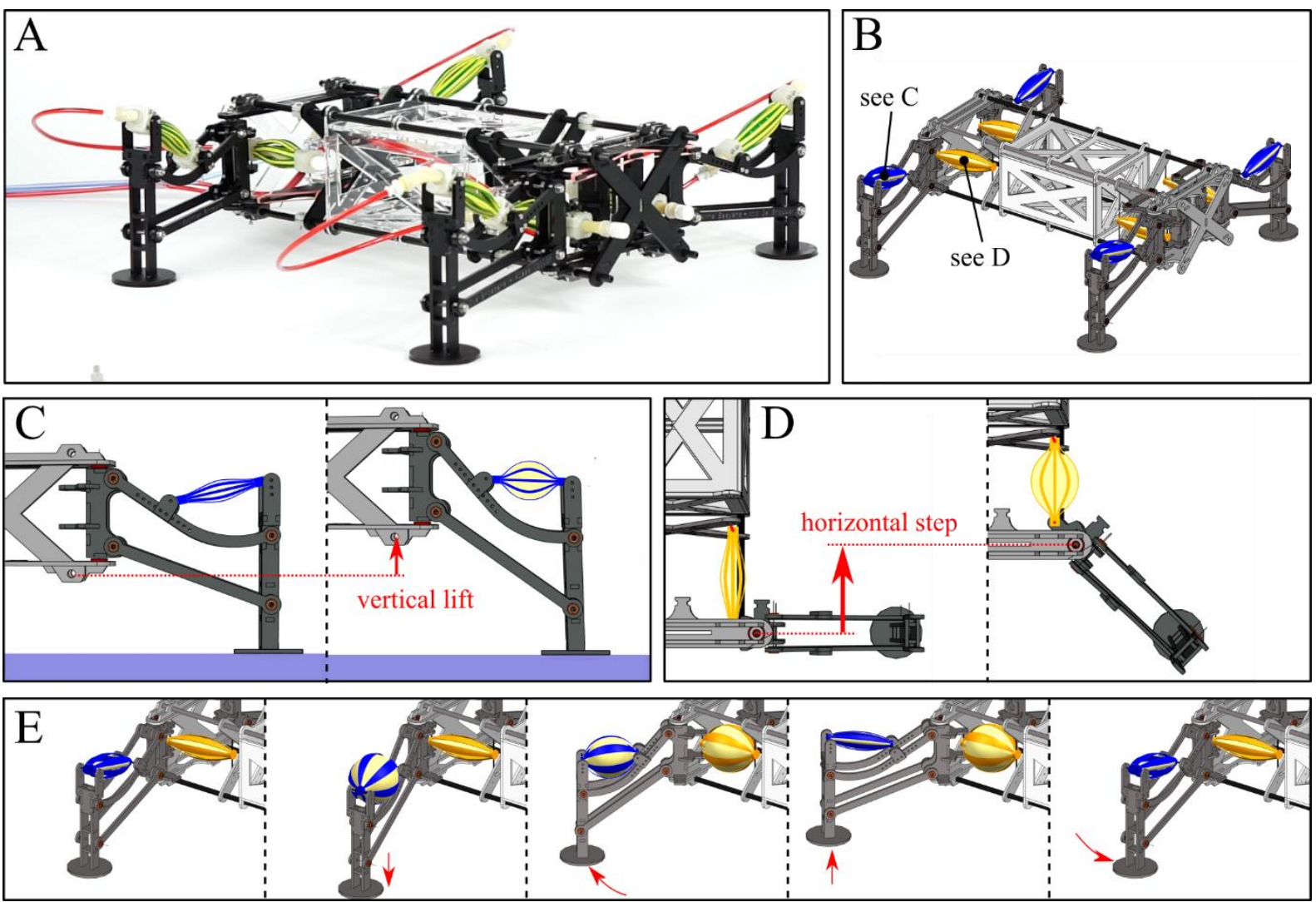

Figure 6 (A,B) Overview picture of the developed tetrapod robot, where each leg is powered using two nonlinear actuators. (C) The blue colored actuators induce a vertical lift of the robot body. (D) The orange colored actuators drive the robot forward. (E) By sequentially actuating the blue and orange actuators in 12|12, a stepping motion is generated.

In the most straightforward tethering, the four different legs of the robot are individually addressed using four separate solenoid valves (see connection scheme in figure S5). Using this wiring, each leg is sequenced in 12|12, effectively resulting in the following actuator sequences: 
$12|12,34| 34,56|56,78| 78$ (actuator number according to figure S4D). By actuating crossed legs in phase with each other, and in antiphase with the other two legs, trotting locomotion is observed, as can be seen on Figure 7A and in Movie S3-S5. The number of tethers can be further reduced to two, by connecting each pair of crossed legs with the same valve (see connection scheme in figure S5). In essence, each leg is still sequenced in 12|12, and by placing a large pressure restriction between crossed legs, an exchange in fluid between both legs is severely restricted. This type of locomotion is shown in Movie S6. A final reduction to only one tether is possible by adding passive supports on the bottom of the robot. The robot rests on these supports while the orange actuators perform their back stroke, which is depicted on figure S6 (see connection scheme in figure S5). This crawling locomotion with only one tether is shown on figure 7B and in Movie S7

The robot weighs $1.1 \mathrm{~kg}$, is able to travel at a speed of 3 body lengths per minute (four tethers) and can carry a maximum pay load of $0.8 \mathrm{~kg}$ (Movie S8). More importantly, the induced hardware sequencing embedded in this robot, allows for walking without extensive tethering (Fig 1A), or on-board valves or control electronics (Fig1B). By directly encoding the actuator sequence in the robots hardware, we have eliminated the need of software control, showing an opportunity for hardware intelligence to be embedded in the nonlinear material characteristics of soft robots.

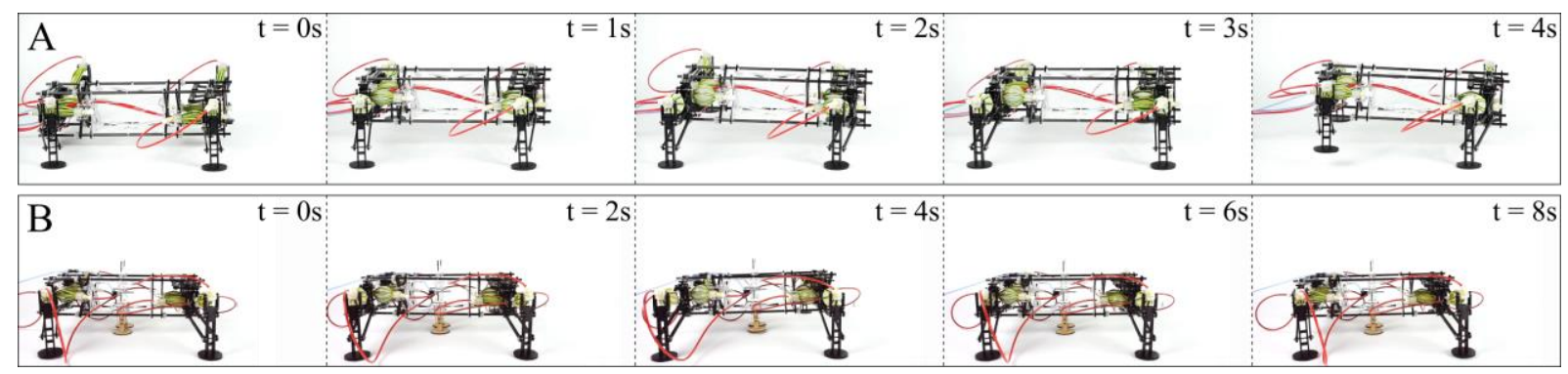

Figure 7. Movie still takes the tetrapod robot for different modes of locomotion: (A) walking with four tethers and (B) crawling with one tether. Images taken from Movie S4 and Movie S7. 


\section{Acknowledgements}

The authors would like to thank N. Govaerts, S. Kushwaha, Q. Brosens and V. De Brouwer for their contributions to this work. This research was supported by the Fund for Scientific Research-Flanders (FWO) and the European Research Council (ERC starting grant HIENA).

Received: ((will be filled in by the editorial staff))

Revised: ((will be filled in by the editorial staff)) Published online: ((will be filled in by the editorial staff))

\section{References}

[1] D. Rus, M. T. Tolley, Nature 2015, 521, 467.

[2] R. Deimel, O. Brock, Int. J. Robotics Res. 2016, 35, 161.

[3] A. De Greef, P. Lambert, A. Delchambre, Precis. Eng. 2009, 33, 311.

[4] E. Brown, N. Rodenberg, J. Amend, A. Mozeika, E. Steltz, M. R. Zakin, H. Lipson, H. M. Jaeger, PNAS 2010, 107, 18809.

[5] S. M. Mirvakili, I. W. Hunter, Adv. Mater. 2017, 30, 1704407.

[6] S. Jun, C. Vito, F. Dario, S. Herbert, Adv. Mater. 2018, 0, 1707035.

[7] M. Ma, L. Guo, D. G. Anderson, R. Langer, Science 2013, 339, 186.

[8] S. Taccola, F. Greco, E. Sinibaldi, A. Mondini, B. Mazzolai, V. Mattoli, Adv. Mater. 2015, 27, 1668.

[9] M. Acerce, E. K. Akdoğan, M. Chhowalla, Nature 2017, 549, 370.

[10] B. Gorissen, D. Reynaerts, S. Konishi, K. Yoshida, J.-W. Kim, M. De Volder, Adv. Mater. 2017, 29, 1604977.

[11] R. V. Martinez, C. R. Fish, X. Chen, G. M. Whitesides, Adv. Funct. Mater. 2012, 22 , 1376.

[12] C. Moraes, Y. Sun, C. A. Simmons, J. Micromech. Microeng. 2009, 19, 065015.

[13] Y. C. Su, L. W. Lin, A. P. Pisano, J. Microelectromech. Syst. 2002, 11, 736.

[14] X. Gong, K. Yang, J. Xie, Y. Wang, P. Kulkarni, A. S. Hobbs, A. D. Mazzeo, Adv. Mater. 2016, 28, 7533.

[15] M. C. Birch, R. D. Quinn, G. Hahm, S. M. Phillips, B. Drennan, A. Fife, H. Verma, R. D. Beer, in Proc. IEEE Int. Conf. on Robotics and Automation (ICRA) 2000, 1109.

[16] T. Chishiro, T. Ono, S. Konishi, in Proc. IEEE Int. Conf. on Micro Electro Mechanical Systems (MEMS) 2013, 532.

[17] K. Takemura, S. Yokota, K. Edamura, in Proc. IEEE Int. Conf. on Robotics and Automation (ICRA) 2005, 532.

[18] B. Verrelst, R. Van Ham, B. Vanderborght, F. Daerden, D. Lefeber, J. Vermeulen, Auton. Rob. 2005, 18, 201.

[19] K. Suzumori, Trans. Jpn. Soc. Mech. Eng. 1989, 55, 2547.

[20] W. Choi, M. Akbarian, V. Rubtsov, C.-J. Kim, IEEE Trans. Ind. Electron. 2009, 56, 1005. 
[21] A. Yamaguchi, K. Takemura, S. Yokota, K. Edamura, Sens. Actuators, A 2011, 170, 139. [22] N. Correll, Ç. D. Önal, H. Liang, E. Schoenfeld, D. Rus, in Proc. Int. Symp. On Exp. Rob. (ISER) 2014, 227.

[23] B. Gorissen, T. Chishiro, S. Shimomura, D. Reynaerts, M. De Volder, S. Konishi, Sens. Actuators, A 2014, 216, 426.

[24] F. Connolly, P. Polygerinos, C. J. Walsh, K. Bertoldi, Soft Rob. 2015, 2, 26.

[25] S. Hirai, T. Masui, S. Kawamura, in Proc. IEEE Int. Conf. on Robotics and Automation (ICRA) 2001, 3807.

[26] A. A. M. Faudzi, R. F. Surakusumah, D. E. Octorina Dewi, I. N. A. Mohd. Nordin, M. R. Muhammad Razif, in Medical Imaging Technology: Reviews and Computational Applications 2015, 223.

[27] K. Ikuta, H. Ichikawa, K. Suzuki, D. Yajima, in Proc. IEEE Int. Conf. on Robotics and Automation (ICRA) 2006, 4161.

[28] M. Cianchetti, T. Ranzani, G. Gerboni, T. Nanayakkara, K. Althoefer, P. Dasgupta, A. Menciassi, Soft Rob. 2014, 1, 122.

[29] Y. Watanabe, M. Maeda, N. Yaji, R. Nakamura, H. Iseki, M. Yamato, T. Okano, S. Hori, S. Konishi, in Proc. IEEE Int. Conf. on Micro Electro Mechanical Systems (MEMS) 2007, 494.

[30] P. Polygerinos, Z. Wang, K. C. Galloway, R. J. Wood, C. J. Walsh, Rob. Auton. Syst. 2015, 73, 135.

[31] Y. Hwang, O. H. Paydar, R. N. Candler, Sens. Actuators, A 2015, 234, 65.

[32] K. C. Galloway, K. P. Becker, B. Phillips, J. Kirby, S. Licht, D. Tchernov, R. J. Wood, D. F. Gruber, Soft Rob. 2016, 3, 23.

[33] K. Suzumori, A. Koga, H. Riyoko, in Proc. IEEE Int. Conf. on Micro Electro Mechanical Systems (MEMS) 1994, 136.

[34] R. K. Katzschmann, A. D. Marchese, D. Rus, in Proc. Int. Symp. On Exp. Rob. (ISER) 2014.

[35] R. F. Shepherd, F. Ilievski, W. Choi, S. A. Morin, A. A. Stokes, A. D. Mazzeo, X. Chen, M. Wang, G. M. Whitesides, PNAS 2011, 108, 20400.

[36] M. T. Tolley, R. F. Shepherd, M. Karpelson, N. W. Bartlett, K. C. Galloway, M. Wehner, R. Nunes, G. M. Whitesides, R. J. Wood, in IEEE/RSJ Int. Conf. on Intelligent Robots and Systems (IROS) 2014, 561.

[37] J.-W. Kim, T. Suzuki, S. Yokota, K. Edamura, Sens. Actuators, A 2012, 174, 155.

[38] K. Suzumori, A. Wada, S. Wakimoto, Sens. Actuators, A 2013, 201, 148.

[39] A. J. M. Moers, M. F. L. De Volder, D. Reynaerts, Biomed. Microdevices 2012, 14, 699.

[40] M. Wehner, R. L. Truby, D. J. Fitzgerald, B. Mosadegh, G. M. Whitesides, J. A. Lewis, R. J. Wood, Nature 2016, 536, 451.

[41] D. Han, H. Gu, J.-W. Kim, S. Yokota, Sens. Actuators, A 2017, 257, 47.

[42] A. A. Stokes, R. F. Shepherd, S. A. Morin, F. Ilievski, G. M. Whitesides, Soft Rob. 2014, 1,70 .

[43] A. D. Marchese, C. D. Onal, D. Rus, Soft Rob. 2014, 1, 75. 
[44] K. Ikuta, H. Ichikawa, K. Suzuki, Medical Image Computing and Computer-Assisted Intervention-(Miccai) 2002, 182.

[45] I. Muller, P. Strehlow, Rubber and rubber balloons: paradigms of thermodynamics 2004.

[46] J. T. B. Overvelde, T. Kloek, J. J. A. D'Haen, K. Bertoldi, PNAS 2015, 112, 10863.

[47] L. Belding, B. Baytekin, H. T. Baytekin, P. Rothemund, M. S. Verma, A. Nemiroski, D. Sameoto, B. A. Grzybowski, G. M. Whitesides, Adv. Mater. 2018, $30,9$.

[48] E. Milana, B. Gorissen, M. De Volder, D. Reynaerts, in IEEE Int. Conf. on Soft Rob. (RoboSoft) 2018, 108.

[49] B. R. Munson, D. F. Young, T. H. Okiishi, Fundamentals of fluid mechanics, Seventh edition 2013. 